\title{
Tunable Kondo effect in graphene with defects
}

\author{
Jian-Hao Chen ${ }^{1,2 \dagger}$, Liang Li ${ }^{2}$, William G. Cullen ${ }^{1,2}$, Ellen D. Williams ${ }^{1,2}$ and Michael S. Fuhrer ${ }^{1,2 \star}$
}

Graphene is a model system for the study of electrons confined to a strictly two-dimensional layer ${ }^{1}$ and a large number of electronic phenomena have been demonstrated in graphene, from the fractional ${ }^{2,3}$ quantum Hall effect to superconductivity4. However, the coupling of conduction electrons to local magnetic moments ${ }^{5,6}$, a central problem of condensed-matter physics, has not been realized in graphene, and, given carbon's lack of $d$ or $f$ electrons, magnetism in graphene would seem unlikely. Nonetheless, magnetism in graphitic carbon in the absence of transition-metal elements has been reported ${ }^{7-9}$, with explanations ranging from lattice defects ${ }^{10}$ to edge structures ${ }^{11}$ to negative curvature regions of the graphene sheet ${ }^{12}$. Recent experiments suggest that correlated defects in highly-ordered pyrolytic graphite (HOPG), induced by proton irradiation ${ }^{8}$ or native to grain boundaries ${ }^{7}$, can give rise to ferromagnetism. Here we show that point defects (vacancies) in graphene ${ }^{13}$ are local moments which interact strongly with the conduction electrons through the Kondo effect ${ }^{6,14-16}$, providing strong evidence that defects in graphene are indeed magnetic. The Kondo temperature $T_{K}$ is tunable with carrier density from 30 to $90 \mathrm{~K}$; the high $T_{K}$ is a direct consequence of strong coupling of defects to conduction electrons in a Dirac material ${ }^{16}$.

We previously reported the resistivity of graphene with vacancies induced by ion irradiation in ultra-high vacuum (UHV; ref. 13). Here we present a detailed study of the gate voltage $\left(V_{\mathrm{g}}\right)$ and temperature $(T)$ dependence of the resistivity $\rho\left(V_{\mathrm{g}}, T\right)$ in similar graphene with vacancies over a wider temperature range $300 \mathrm{mK}<T<290 \mathrm{~K}$. Apart from weak-localization (WL) corrections $^{17,18}$, we find that $\rho\left(V_{\mathrm{g}}, T\right)$ is explained by a temperatureindependent contribution $\rho_{c}\left(V_{\mathrm{g}}\right)$ due to non-magnetic disorder plus a temperature-dependent contribution $\rho_{\mathrm{K}}\left(V_{\mathrm{g}}, T\right)$, not present in as-prepared graphene ${ }^{13}$, which follows the universal temperature dependence expected for Kondo scattering from a localized $1 / 2$ spin with a single scaling parameter $T_{\mathrm{K}}$.

Graphene with vacancies is prepared as described in ref. 13. After irradiation, the devices were annealed overnight at $490 \mathrm{~K}$ in $\mathrm{UHV}$, and then exposed to air during transfer to a ${ }^{3} \mathrm{He}$ samplein-vacuum cryostat. Figure la shows $\sigma\left(V_{\mathrm{g}}\right)$ measured at $17 \mathrm{~K}$ for a graphene device (sample Q6) before irradiation, immediately after irradiation, and measured at $300 \mathrm{mK}$ after annealing and transfer to the ${ }^{3} \mathrm{He}$ cryostat. $V_{\mathrm{g}}$ is applied to the $\mathrm{Si}$ substrate to tune the carrier density $n=c_{g} V_{\mathrm{g}} / e$, where $c_{g}=1.15 \times 10^{-8} \mathrm{~F} \mathrm{~cm}^{-2}$ is the gate capacitance, and $e$ the elementary charge. The mobility of the device is approximately 4000,300 , and $2000 \mathrm{~cm}^{2} \mathrm{~V}^{-1} \mathrm{~s}^{-1}$, respectively, for these three measurements; the conductivity and mobility recover significantly after annealing and air exposure, consistent with our previous study ${ }^{13}$. From the post-annealing mobility we estimate that this device has a defect density, $n_{\mathrm{imp}}$, of approximately $3 \times 10^{11} \mathrm{~cm}^{-2}$, although greater understanding of the effects of annealing and ambient exposure on vacancies in graphene is needed. See Supplementary Information for the calculation of defect density and also Raman spectra of the device before and after irradiation. Slight asymmetry between electron and hole conduction in the $\sigma\left(V_{\mathrm{g}}\right)$ curve is also observed in the irradiated sample, which could indicate a non-zero on-site energy for the defects in graphene ${ }^{19}$.

Figure $1 \mathrm{~b}$ shows the perpendicular magnetic field dependence of the resistivity $\rho(B)$ of the irradiated sample Q6 at $T=300 \mathrm{mK}$ at several different gate voltages. Negative magnetoresistance is observed at small $B$, indicating the dominance of weak localization arising from intervalley scattering due to lattice defects ${ }^{17,18}$. Figure 1c shows a detail of the magnetoresistance at small $B$, at $300 \mathrm{mK}$ and at $V_{\mathrm{g}}-V_{\mathrm{g}, \min }=-65 \mathrm{~V}$ (see Supplementary Information for the gate voltage and temperature dependent phase coherence length, which is extracted from analyzing the WL magnetoresistance). Shubnikov-de Haas ( $\mathrm{SdH})$ oscillations appear at high $B$ field. To measure the resistivity without WL and $\mathrm{SdH}$ corrections, the WL contribution is suppressed by application of $B=1 \mathrm{~T}$ in further measurements. For $\left|V_{\mathrm{g}}-V_{\mathrm{g}, \min }\right|<5 \mathrm{~V}$, $\mathrm{SdH}$ corrections may affect the data slightly at $1 \mathrm{~T}$. However, as shown below, the $\rho(T)$ behaviour for $\left|V_{\mathrm{g}}-V_{\mathrm{g}, \min }\right|>5 \mathrm{~V}$ and $\left|V_{\mathrm{g}}-V_{\mathrm{g}, \min }\right|<5 \mathrm{~V}$ show no qualitative differences.

Figure 2 a shows the temperature-dependent resistivity $\rho(T)$ of the irradiated graphene measured at several different gate voltages at $B=1 \mathrm{~T}$. Positive slopes, $\mathrm{d} \rho / \mathrm{d} T>0$, are seen in $\rho(T)$ from room temperature to about $200 \mathrm{~K}$ for $V_{\mathrm{g}}$ not too near $V_{\mathrm{g}, \mathrm{min}}$, indicating phonon contributions ${ }^{20}$; between $\sim 10$ and $\sim 100 \mathrm{~K}$, we find $\mathrm{d} \rho / \mathrm{d} T<0$ and the resistivity increases logarithmically with decreasing temperature at all $V_{\mathrm{g}}$. At low temperature the resistivity at all $V_{\mathrm{g}}$ saturates $(\mathrm{d} \rho / \mathrm{d} T \rightarrow 0)$, indicating that there is no disorder-induced metal to insulator transition (MIT; ref. 16) or opening of a bandgap.

In metallic systems where localized magnetic moments couple anti-ferromagnetically to the conduction electrons, spin-flip scattering gives rise to an anomalous component of the resistivity $\rho_{\mathrm{K}}(T)$ which is characterized by a Kondo temperature $T_{\mathrm{K}}$ (refs 6,21). For $T \approx T_{\mathrm{K}}, \rho_{\mathrm{K}}(T)$ is approximately logarithmic in $T$ (similar behaviour is observed in situ in UHV before ambient exposure; see Supplementary Information). In principle, interaction effects in the presence of disorder could also lead to logarithmic $\rho(T)$ even at high magnetic field (the Altshuler-Aronov effect); however this would also lead to similar corrections to the Hall resistivity, which are not observed (see Supplementary Information). For $T \ll T_{\mathrm{K}}$, the conduction electrons screen the spins of the local moments and the resistivity saturates, with a negative correction proportional to $T^{2}$ (ref. 22). To compare the observed data in graphene with vacancies to theories of the Kondo effect, we model the

\footnotetext{
${ }^{1}$ Materials Research Science and Engineering Center, University of Maryland, College Park, Maryland 20742, USA, ${ }^{2}$ Center for Nanophysics and Advanced Materials, Department of Physics, University of Maryland, College Park, Maryland 20742, USA. †Present address: Department of Physics and Center of Integrated Nanomechanical Systems, University of California at Berkeley, Berkeley, California 94720, USA; Materials Sciences Division, Lawrence Berkeley National Laboratory, Berkeley, California 94720, USA. *e-mail:mfuhrer@umd.edu.
} 

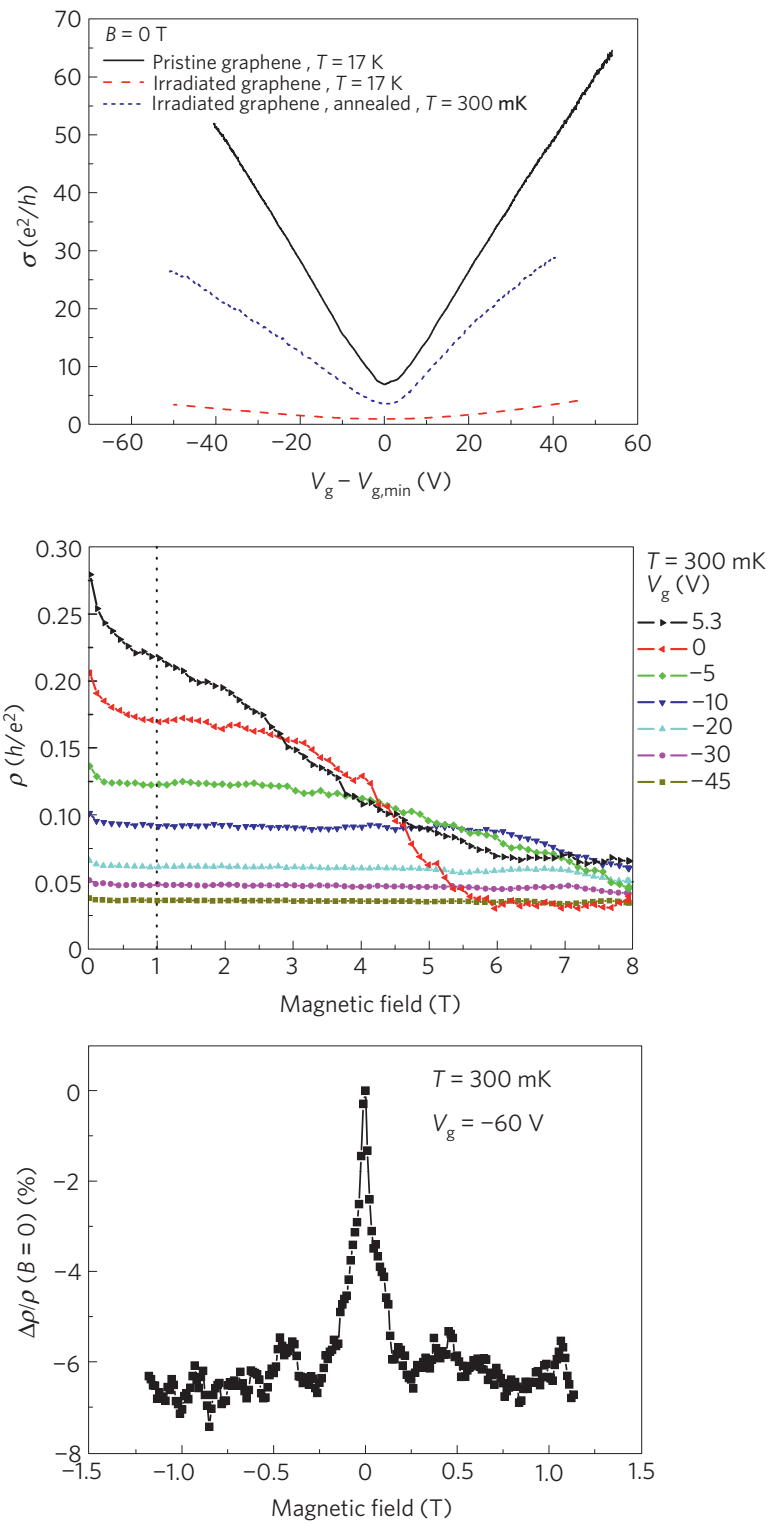

Figure 1 | Gate voltage dependent conductivity $\sigma\left(V_{g}\right)$ and magnetoresistance of the graphene sample. $\mathbf{a}, \sigma\left(V_{g}\right)$ of the graphene sample Q6 before (black solid line) and after (red dashed line) irradiation with $500 \mathrm{eV} \mathrm{He}^{+}$at a temperature $T=17 \mathrm{~K}$, and after annealing at $490 \mathrm{~K}$ overnight in ultra-high vacuum and exposure to ambient before cooling to $T=300 \mathrm{mK}$ (blue short-dashed line). Magnetic field $B=0$ for all data. The gate voltage of minimum conductivity $V_{g, \min }=-8 \mathrm{~V}, 5 \mathrm{~V}, 5.3 \mathrm{~V}$ for pristine, irradiated and annealed sample, respectively. $\mathbf{b}$, Magnetoresistance of irradiated and annealed graphene sample for $B=0-8 T$ at various $V_{g}$. c, Normalized detailed magnetoresistance of irradiated and annealed graphene sample from -1.2 to $1.2 \mathrm{~T}$ at $V_{\mathrm{g}}-V_{\mathrm{g}, \min } \approx-65 \mathrm{~V}$.

temperature-dependent resistivity in the low temperature regime and the intermediate temperature regime (region of maximum logarithmic slope, roughly between 10 and $100 \mathrm{~K}$ ), respectively, as

$$
\begin{gathered}
\rho\left(V_{\mathrm{g}}, T\right)=\rho_{c 1}\left(V_{\mathrm{g}}\right)+\rho_{\mathrm{K}, 0}\left(V_{\mathrm{g}}\right)\left(1-\left(\frac{\pi}{2}\right)^{4}\left(\frac{T}{T_{\mathrm{K}}\left(V_{\mathrm{g}}\right)}\right)^{2}\right) \\
\rho\left(V_{\mathrm{g}}, T\right)=\rho_{c 2}\left(V_{\mathrm{g}}\right)+\frac{\rho_{\mathrm{K}, 0}\left(V_{\mathrm{g}}\right)}{2}\left(1-0.470 \ln \left(\frac{1.2 T}{T_{\mathrm{K}}\left(V_{\mathrm{g}}\right)}\right)\right)
\end{gathered}
$$

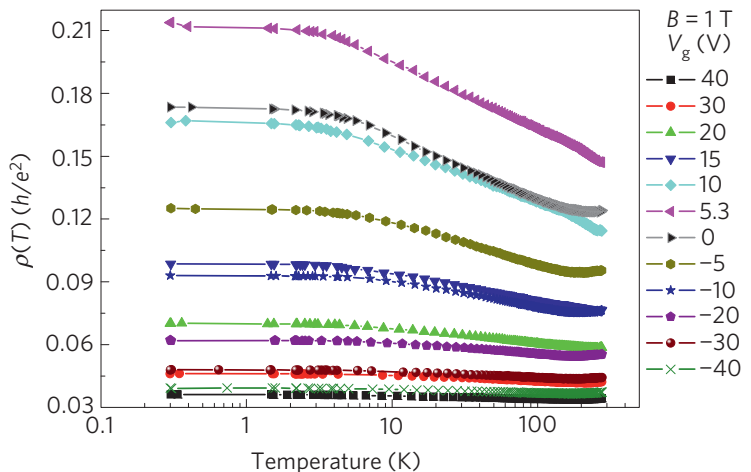

b

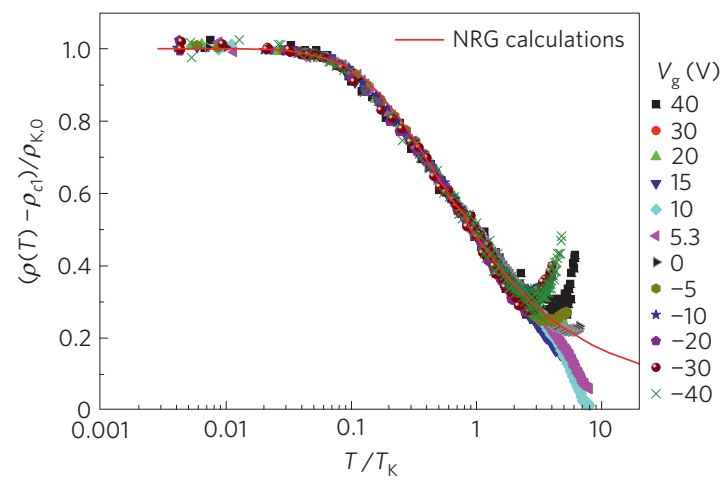

Figure 2 | Universal Kondo behaviour of graphene with defects.

a, Temperature-dependent resistivity $\rho\left(V_{\mathrm{g}}\right)$ of graphene sample Q6 under a perpendicular magnetic field of $1 \mathrm{~T}$, at 12 different gate voltages, with temperature changing from $300 \mathrm{mK}$ to $\sim 290 \mathrm{~K}$. b. The normalized Kondo part of the resistivity $\left(\rho-\rho_{c 1}\right) / \rho_{K}, 0$ versus $T / T_{K}\left(V_{g}\right)$, where $T_{K}\left(V_{g}\right)$ is the Kondo temperature at respective gate voltage (see Fig. 4 ). The red line is the expected universal Kondo behaviour from numerical renormalization group calculations ${ }^{21}$.

where $\rho_{\mathrm{K}, 0}$ is the Kondo resistivity at zero temperature, $\rho_{c 1}$ and $\rho_{c 2}$ the non-temperature-dependent part of the resistivity, presumably from impurity scattering that does not involve the spin degree of freedom $^{23,24}$. The numerical factors in equations (1) and (2) are from the theory of the spin-1/2 Kondo effect ${ }^{21}$. As $\rho\left(V_{\mathrm{g}}, T=0\right)$ is known, there are three degrees of freedom in these equations at each $V_{\mathrm{g}}: \rho_{c 1}, \rho_{c 2}$ and $T_{\mathrm{K}}$; if $\rho_{\mathrm{K}}(T)$ follows the universal Kondo form then $\rho_{c 1}=\rho_{c 2}$. We keep $\rho_{c 1}$ and $\rho_{c 2}$ as independent parameters to test the internal consistency of the model. Least square fits to the equations (1) and (2) are carried out on $\rho\left(V_{\mathrm{g}}, T\right)$ in the low and intermediate temperature ranges respectively (see Supplementary Information for details).

Using the extracted parameters, we can scale the $\rho(T)$ curves at different $V_{\mathrm{g}}$ and compare them to the universal Kondo behaviour ${ }^{21,25}$. Figure $2 \mathrm{~b}$ shows the normalized Kondo resistivity $\left(\rho-\rho_{c 1}\right) / \rho_{\mathrm{K}, 0}$ versus $T / T_{\mathrm{K}}$ and the universal Kondo behaviour from numerical renormalization group calculations (NRG; ref. 21). From Fig. 2b one can find that: (1) all the experimental curves collapse to a single functional form for $300 \mathrm{mK}<T<\sim 3 T_{\mathrm{K}}$ and (2) the functional form matches well the universal Kondo behaviour from NRG calculations. At higher temperature $(T>200 \mathrm{~K})$, phonon contributions become important ${ }^{20}$ and the observed positive deviations from the NRG calculations are expected. However, at the lowest gate voltages, the deviation is negative, possibly due to thermal activation of carriers.

Now we discuss the gate voltage dependence of the extracted parameters, $\rho_{c 1}, \rho_{c 2}, \rho_{\mathrm{K}, 0}$ and $T_{\mathrm{K}}$. Figure $3 \mathrm{a}$ shows $\rho_{c 1}$ and $\rho_{c 2}$ versus $V_{\mathrm{g}}$, which peak around the actual minimum conductivity gate voltage $V_{\mathrm{g}, \min } \approx 5.3 \mathrm{~V}$. We find that $\rho_{c 1}$ and $\rho_{c 2}$ are practically 
a

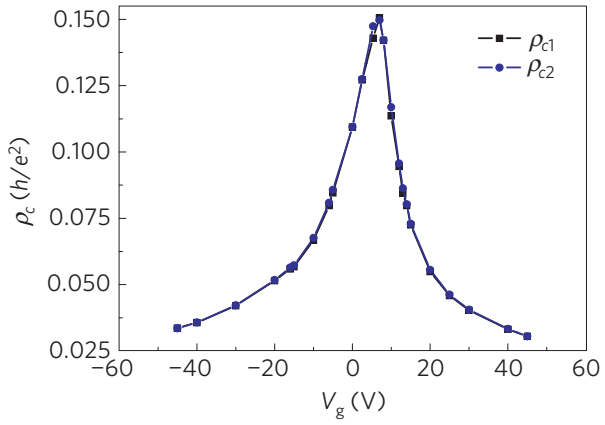

c

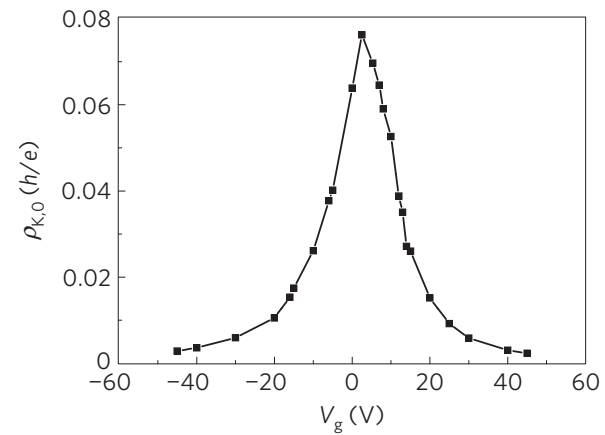

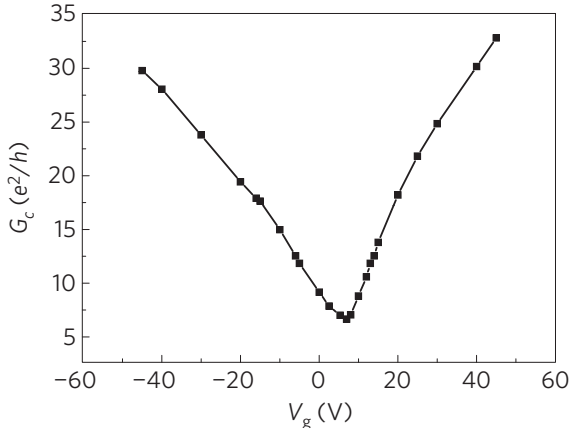

d

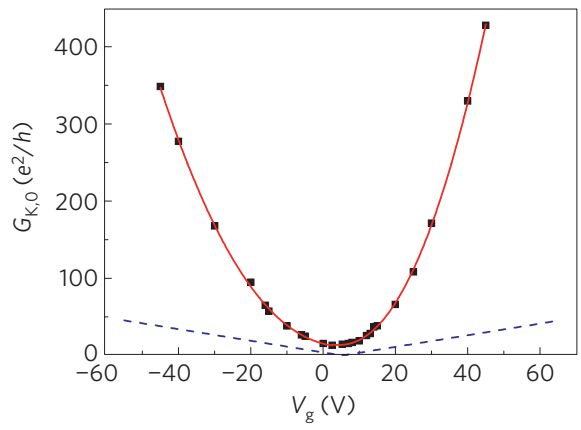

Figure $\mathbf{3}$ | The non-Kondo and Kondo part of the resistivity versus $\boldsymbol{V}_{\mathbf{g}} \mathbf{a}$, Comparison between the non-Kondo resistivity obtained from fitting $\rho\left(V_{\mathrm{g}}, T\right)$ to equation (1) in the low temperature regime $\left(\rho_{c 1}\right)$ and to equation (2) in the intermediate temperature regime $\left(\rho_{c 2}\right)$ at different $V_{g}$. b, non-Kondo conductivity $G_{c}=1 / \rho_{c}$ as a function of $V_{g}$. c, The zero temperature Kondo resistivity $\rho_{K, 0}$ and $\mathbf{d}$, Kondo conductivity $G_{K, 0}=1 / \rho_{K, 0}$ as a function of $V_{g}$. The red solid line in $\mathbf{d}$ is a power law fit to $G_{K, 0},\left(V_{g}\right)$. The blue dashed line is the expectation for unitary scatterers of concentration $3 \times 10^{11} \mathrm{~cm}^{-2}\left(r^{2} f .15\right)$.

identical, which indicates that the logarithmic divergence and $T^{2}$ saturation of the resistivity indeed arise from the same effect (the Kondo effect). From now on we use $\rho_{c 1}$ for the non-Kondo resistivity and label it as $\rho_{c}$. Figure $3 \mathrm{~b}$ shows the non-Kondo conductivity $G_{c}=1 / \rho_{c}$ as a function of $V_{\mathrm{g}}$, which has a similar gate voltage dependence as an as-prepared graphene sample. That is linear $G\left(V_{\mathrm{g}}\right)$ at high $V_{\mathrm{g}}$, and a minimum $G$ of a few $e^{2} / h$ where $h$ is Planck's constant. It is worth noting that the minimum nonKondo conductivity $G_{c, \min }=6.9 e^{2} / h$ is the same as the minimum conductivity of the pristine sample before irradiation (see Fig. 1a).

Figure $3 \mathrm{c}$ shows the Kondo resistivity $\rho_{\mathrm{K}, 0}=\rho(T=0)-\rho_{c}$ versus $V_{\mathrm{g}}$, which also peaks around the $V_{\mathrm{g}, \min }$, and decreases rapidly with increasing $\left|V_{\mathrm{g}}-V_{\mathrm{g}, \min }\right|$, and Fig. $3 \mathrm{~d}$ shows $G_{\mathrm{K}, 0}=1 / \rho_{\mathrm{K}, 0}$ versus $V_{\mathrm{g}}$. In the low-temperature limit (saturated resistivity), we expect $G_{\mathrm{K}, 0} \approx\left(\pi e^{2} / h\right)\left(n / n_{\mathrm{imp}}\right)$ (ref. 15). However, $G_{\mathrm{K}, 0}$ is $3-10$ times larger than expected for $n_{\text {imp }}=3 \times 10^{11} \mathrm{~cm}^{-2}$, and varies more rapidly; the red solid line is a power law fit to $G_{\mathrm{K}, 0}\left(V_{\mathrm{g}}\right)$ that yields $G_{\mathrm{K}, 0} \sim A+B V_{\mathrm{g}}^{\alpha}$, with $\alpha=2.1 \pm 0.1$ for electron conduction and $\alpha=2.2 \pm 0.2$ for hole conduction.

Figure 4 shows the gate voltage dependence of the Kondo temperature $T_{\mathrm{K}} . T_{\mathrm{K}}$ is of order $50 \mathrm{~K}$, which indicates strong coupling between the localized magnetic moment and the conduction electrons. Moreover, $T_{\mathrm{K}}$ is tunable by gate voltage, with a minimum of about $30 \mathrm{~K}$ near $V_{\mathrm{g} \text {,min }}$ and maxima close to $90 \mathrm{~K}$ at $V_{\mathrm{g}}-V_{\mathrm{g}, \min } \approx-20 \mathrm{~V}$ (hole conduction) and close to $70 \mathrm{~K}$ at $V_{\mathrm{g}}-V_{\mathrm{g}, \min } \approx 25 \mathrm{~V}$ (electron conduction). At higher gate voltages $\left(\left|V_{\mathrm{g}}-V_{\mathrm{g}, \min }\right|>25 \mathrm{~V}\right), T_{\mathrm{K}}$ decreases slightly with gate voltage, although the experimental error becomes large at large $V_{\mathrm{g}}$, as the Kondo resistivity become very small. $T_{\mathrm{K}}$ versus $V_{\mathrm{g}}-V_{\mathrm{g}, \min }$ for a second sample (sample L2, see Supplementary Information for details) shows similar magnitude and similar variation with gate voltage. The higher base measurement temperature $(1.7 \mathrm{~K}$ for sample $\mathrm{L} 2$ as compare to $0.3 \mathrm{~K}$ for sample discussed in main text) results in larger uncertainty in $T_{\mathrm{K}}$, and might result in a systematically smaller $T_{\mathrm{K}}$ obtained from the fitting. It is also possible that $T_{\mathrm{K}}$ depends on the initial

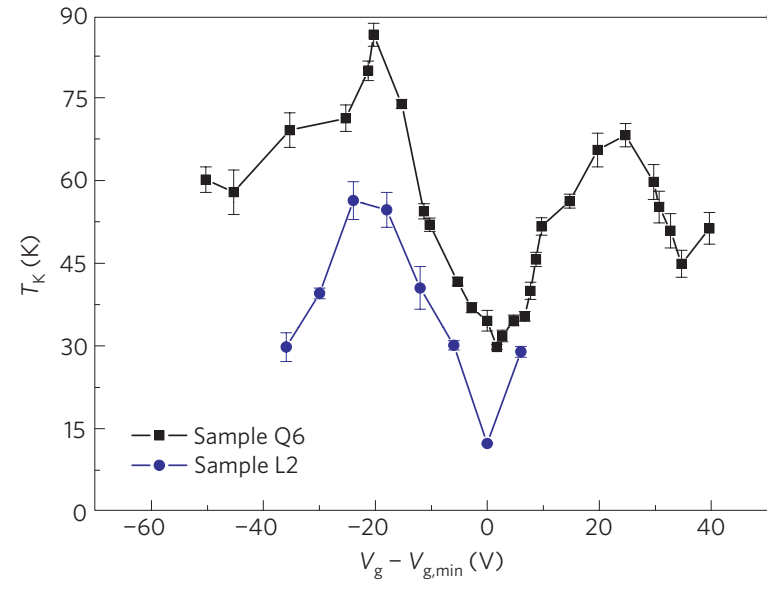

Figure 4 | The gate-tunable Kondo Temperature. The Kondo temperature $T_{K}$ of two graphene samples with vacancies as a function of gate voltage $V_{g}$ as determined from fits to equations (1) and (2). The sample Q6 (black squares) has been discussed in the main text. Details on sample L2 (blue circles) can be found in Supplementary Information. The error bars represent the \pm one standard deviation of $T_{\mathrm{K}}$, calculated using error propagation from the standard deviation of raw fitting parameters (see Supplementary Information).

disorder in the sample, as electron-hole puddling may play a role in determining $T_{\mathrm{K}}$.

In a conventional (non-relativistic) Fermi liquid, we can roughly estimate the Kondo temperature:

$$
k_{\mathrm{B}} T_{\mathrm{K}} \approx D \mathrm{e}^{-1 / J \rho\left(E_{\mathrm{F}}\right)}
$$

where $k_{\mathrm{B}}$ is Boltzmann's constant, $D$ is the electronic bandwidth ( $\sim 10 \mathrm{eV}$ for $\pi$ electrons in graphene), $J>0$ the antiferromagnetic coupling constant, and $\rho\left(E_{\mathrm{F}}\right)=8 \pi E_{\mathrm{F}} /\left(h v_{\mathrm{F}}\right)^{2}=4 \sqrt{\pi n} / h \nu_{\mathrm{F}}$ is the 
density of states of graphene at the Fermi energy. Equation (3) predicts $T_{\mathrm{K}}$ vanishes exponentially as $E_{\mathrm{F}} \rightarrow 0$. This is in contrast to the observed variation of only $\sim 3 \times$ in $T_{K}$. The Kondo effect in a relativistic electron system is expected to differ qualitatively from the conventional non-relativistic Kondo effect ${ }^{14,26,27}$. In particular, for systems with $\rho\left(E_{\mathrm{F}}\right) \propto\left|E_{\mathrm{F}}\right|^{r}$, the case of $r=1$ (expected for graphene) is critical, and $T_{\mathrm{K}}$ vanishes as $E_{\mathrm{F}} \rightarrow 0$ either linearly or as a power law, depending on the sign of $E_{\mathrm{F}}$. For $r>1, T_{\mathrm{K}}$ remains finite, and for $r<1, T_{\mathrm{K}}$ is exponentially suppressed, as $E_{\mathrm{F}} \rightarrow 0$. A recent theoretical study ${ }^{27}$ of (AB sublattice symmetric) Co on graphene found large $T_{\mathrm{K}}$, of order $100 \mathrm{~K}$, and strong asymmetry in $T_{\mathrm{K}}$ about $E_{\mathrm{F}}=0$, which we do not observe. However, the defects studied here are expected to break sublattice symmetry and locally open a gap at the Dirac point, with a sharp resonant midgap state leading to resonant impurity scattering as $E_{\mathrm{F}} \rightarrow 0$ (refs 13,16). In this case, it has been pointed out that the dimensionless parameter which determines the strength of the Kondo effect, $J \rho\left(E_{\mathrm{F}}\right)$ in equation $(3)$, is proportional to the sine of the scattering phase shift for the defect, which for the large scalar potentials induced by defects in the graphene plane is finite and of order unity ${ }^{16}$ as $E_{\mathrm{F}} \rightarrow 0$, leading to a robust Kondo effect. For a resonant state located at exactly the Dirac point we would also expect a strong particle-hole asymmetry as the defect state is filled and emptied for positive or negative $E_{\mathrm{F}}$. However, we suppose that a state located modestly below the Dirac point could be filled at all observable $E_{\mathrm{F}}$ (for $V_{\mathrm{g}}<50 \mathrm{~V}, E_{\mathrm{F}}<240 \mathrm{meV}$ ) and still produce a strongly resonant scattering around $E_{\mathrm{F}}=0$ (ref. 19). It is also probable that in disordered graphene with potential fluctuations ${ }^{28}$, the resistivity probes only the few defects with the largest $T_{\mathrm{K}}$; this is consistent with $G_{\mathrm{K}, 0}$ larger than expected in the low-temperature limit (Fig. 3d). A complete theory of the Kondo effect in graphene will probably require both a microscopic understanding of the defect and its interaction with conduction electrons, as well as an effective medium theory of Kondo scattering in the presence of potential variations in disordered graphene.

The high $T_{\mathrm{K}}$ in graphene with its small density of states is a unique consequence of defect scattering in a Dirac system ${ }^{16}$, and we anticipate that new graphene Kondo systems may be realized by a variety of physical or chemical modifications to the graphene lattice. Defect engineering thus provides a powerful route to introduce and control magnetism in carbon nanostructures, such as graphene and carbon nanotubes, without the presence of transition metal elements. The observation of Kondo scattering from defects in graphene may also explain the anomalous short spin lifetimes observed in graphene spin valves in the currently explored temperature range ${ }^{29}$; as a small native concentration of defects could be present in these (and perhaps all) graphene devices ${ }^{30}$.

Received 29 April 2010; accepted 3 March 2011; published online 3 April 2011

\section{References}

1. Geim, A. K. Graphene: Status and prospects. Science 324, 1530-1534 (2009)

2. Bolotin, K. I., Ghahari, F., Shulman, M. D., Stormer, H. L. \& Kim, P. Observation of the fractional quantum Hall effect in graphene. Nature 462, 196-199 (2009).

3. Du, X., Skachko, I., Duerr, F., Luican, A. \& Andrei, E. Y. Fractional quantum Hall effect and insulating phase of Dirac electrons in graphene. Nature 462, 192-195 (2009).

4. Heersche, H. B., Jarillo-Herrero, P., Oostinga, J. B., Vandersypen, L. M. K. \& Morpurgo, A. F. Bipolar supercurrent in graphene. Nature 446, 56-59 (2007).

5. Anderson, P. W. Localized magnetic states in metals. Phys. Rev. 124, 41-53 (1961).

6. Kondo, J. Resistance minimum in dilute magnetic alloys. Prog. Theor. Phys. 32, 37-49 (1964).
7. Cervenka, J., Katsnelson, M. I. \& Flipse, C. F. J. Room-temperature ferromagnetism in graphite driven by two-dimensional networks of point defects. Nature Phys. 5, 840-844 (2009).

8. Esquinazi, P. et al. Induced magnetic ordering by proton irradiation in graphite Phys. Rev. Lett. 91, 227201 (2003).

9. Ugeda, M. M., Brihuega, I., Guinea, F. \& Gomez-Rodriguez, J. M. Missing atom as a source of carbon magnetism. Phys. Rev. Lett. 104, 096804 (2010).

10. Lehtinen, P. O., Foster, A. S., Ma, Y., Krasheninnikov, A. V. \& Nieminen, R. M. Irradiation-induced magnetism in graphite: A density functional study. Phys. Rev. Lett. 93, 187202 (2004).

11. Fujita, M., Wakabayashi, K., Nakada, K. \& Kusakabe, K. Peculiar localized state at zigzag graphite edge. J. Phys. Soc. Jpn 65, 1920-1923 (1996).

12. Park, N. et al. Magnetism in all-carbon nanostructures with negative Gaussian curvature. Phys. Rev. Lett. 91, 237204 (2003).

13. Chen, J-H., Cullen, W. G., Jang, C., Fuhrer, M. S. \& Williams, E. D. Defect scattering in graphene. Phys. Rev. Lett. 102, 236805 (2009).

14. Sengupta, K. \& Baskaran, G. Tuning Kondo physics in graphene with gate voltage. Phys. Rev. B 77, 045417 (2008).

15. Cornaglia, P. S., Usaj, G. \& Balseiro, C. A. Localized spins on graphene. Phys. Rev. Lett. 102, 046801 (2009).

16. Hentschel, M. \& Guinea, F. Orthogonality catastrophe and Kondo effect in graphene. Phys. Rev. B 76, 115407 (2007).

17. Morpurgo, A. F. \& Guinea, F. Intervalley scattering, long-range disorder, and effective time-reversal symmetry breaking in graphene. Phys. Rev. Lett. 97, 196804 (2006).

18. McCann, E. et al. Weak-localization magnetoresistance and valley symmetry in graphene. Phys. Rev. Lett. 97, 146805 (2006).

19. Wehling, T. O., Yuan, S., Lichtenstein, A. I., Geim, A. K. \& Katsnelson, M. I. Resonant scattering by realistic impurities in graphene. Phys. Rev. Lett. 105, 056802 (2010).

20. Chen, J-H., Jang, C., Xiao, S., Ishigami, M. \& Fuhrer, M. S. Intrinsic and extrinsic performance limits of graphene devices on SiO2. Nature Nanotech. 3, 206-209 (2008).

21. Costi, T. A. et al. Transport coefficients of the Anderson model via the numerical renormalization group. J. Phys. Condens. Matter 6, 2519-2558 (1994).

22. Nozières, P. A Fermi-liquid description of the Kondo problem at low temperatures. J. Low Temp. Phys. 17, 31-42 (1974).

23. Chen, J-H. et al. Charged impurity scattering in graphene. Nature Phys. $\mathbf{4}$ 377-381 (2008)

24. Jang, C. et al. Tuning the effective fine structure constant in graphene: Opposing effects of dielectric screening on short- and long-range potential scattering. Phys. Rev. Lett. 101, 146805 (2008).

25. Goldhaber-Gordon, D. et al. From the Kondo regime to the mixed-valence regime in a single-electron transistor. Phys. Rev. Lett. 81, 5225-5228 (1998).

26. Cassanello, C. R. \& Fradkin, E. Kondo effect in flux phases. Phys. Rev. B 53, 15079-15094 (1996).

27. Vojta, M., Fritz, L. \& Bulla, R. Gate-controlled Kondo screening in graphene: Quantum criticality and electron-hole asymmetry. Europhys. Lett. 90, 27006 (2010).

28. Rossi, E. \& Das Sarma, S. Ground state of graphene in the presence of random charged impurities. Phys. Rev. Lett. 101, 166803 (2008).

29. Popinciuc, M. et al. Electronic spin transport in graphene field-effect transistors. Phys. Rev. B 80, 214427 (2009).

30. Ni, Z. H. et al. On resonant scatterers as a factor limiting carrier mobility in graphene. Nano Lett. 10, 3868-3872 (2010).

\section{Acknowledgements}

This work has been supported by NSF-UMD-MRSEC grant DMR 05-20471 (J-H.C., W.G.C., E.D.W., M.S.F.) and the US ONR grant N000140610882 (W.G.C., E.D.W., M.S.F.). The MRSEC SEFs were used in this work. Infrastructure support has also been provided by the UMD NanoCenter and CNAM. We would also like to thank D. Goldhaber-Gordon, S. D. Sarma, E. Rossi, E. Hwang and J. Zhu for useful discussions.

\section{Author contributions}

J-H.C. and M.S.F. conceived the experiments, J-H.C. and L.L. fabricated devices, J-H.C. performed the experiments and analyzed the data, J-H.C., W.G.C., E.D.W. and M.S.F. co-wrote the paper. All authors discussed the results and commented on the manuscript.

\section{Additional information}

The authors declare no competing financial interests. Supplementary information accompanies this paper on www.nature.com/naturephysics. Reprints and permissions information is available online at http://npg.nature.com/reprintsandpermissions. Correspondence and requests for materials should be addressed to M.S.F. 